\title{
Risk factors for short-term outcomes after thoracoscopic lobectomy for lung cancer
}

\author{
Masataka Irie ${ }^{1}$, Ryoichi Nakanishi ${ }^{2}$, Manabu Yasuda ${ }^{3}, Y_{0}$ shihisa Fujino ${ }^{4}$, \\ Kazumi Hamada ${ }^{1}$ and Masahiro Hyodo ${ }^{1}$
}

Affiliations: ${ }^{1}$ Department of Rehabilitation Medicine, Shin-Kokura Hospital, Federation of National Public Service Personnel Mutual Aid Associations, Kitakyushu, Japan. ${ }^{2}$ Department of Oncology, Immunology and Surgery, Nagoya City University Graduate School of Medical Science and Medical School, Nagoya, Japan. ${ }^{3}$ Department of Thoracic Surgery, Shin-Kokura Hospital, Federation of National Public Service Personnel Mutual Aid Associations, Kitakyushu, Japan. "Department of Preventive Medicine and Community Health, University of Occupational and Environmental Health, Kitakyushu, Japan.

Correspondence: Masataka Irie, Department of Rehabilitation Medicine, Shin-Kokura Hospital, Federation of National Public Service Personnel Mutual Aid Associations, 1-3-1 Kanada, Kokurakita-ku, Kitakyushu, 803-8505, Japan. E-mail: DQL02511 anifty.com

ABSTRACT Few studies have analysed postoperative risk factors in patients undergoing thoracoscopic lobectomy, including assessments of preoperative physical function. The objectives of this study were to identify predictors of postoperative deterioration of performance status and cardiopulmonary complications in cases of thoracoscopic lobectomy.

Between June 2005 and October 2012, we retrospectively reviewed 188 consecutive subjects who underwent thoracoscopic lobectomy for preoperative stage I nonsmall cell lung cancer. The demographic and clinical parameters, including physical function, were analysed using a multivariate logistic regression to clarify the determinants.

The percent predicted diffusing capacity of the lung for carbon monoxide, quadriceps muscle strength and pathologic stage were independent risk factors for deterioration of performance status after surgery in the multivariate analyses. Chronic obstructive pulmonary disease, 6-min walking distance and pathologic stage were also independent risk factors for postoperative cardiopulmonary complications.

Our data suggest that, in addition to a greater pathologic stage, lower diffusing capacity and comorbid chronic obstructive pulmonary disease, poor physical function was associated with worse short-term outcomes after thoracoscopic lobectomy. An evaluation of preoperative quadriceps muscle strength and 6-min walk test is easily performed and may therefore be a useful predictor in cases of thoracoscopic lobectomy.

@ERSpublications

Evaluating quadriceps muscle and 6MWT is useful for risk assessment in cases of thoracoscopic lobectomy for NSCLC http://ow.ly/4mIZQR 


\section{Introduction}

The application of thoracoscopic lobectomy in patients with early-stage nonsmall cell lung cancer (NSCLC) has been spreading, as many published studies have demonstrated that thoracoscopic lobectomy is superior to thoracotomy in terms of perioperative morbidity and a reduced hospital length of stay, with equal long-term outcomes [1-3]. However, few studies have specifically analysed postoperative risk factors only in patients undergoing thoracoscopic lobectomy [4-6], despite considerable comparative studies of a thoracoscopic versus open thoracotomy approach $[1-3,7,8]$. In addition, there is very limited evidence determining whether assessing preoperative physical functioning parameters, such as functional capacity and muscle strength, has predictive value for determining the postoperative outcomes in patients undergoing thoracoscopic lobectomy.

Among the various immediate postoperative outcomes, identifying risk factors regarding a change in the performance status or incidence of complications after surgery is clinically useful because of the potentially negative impact on oncologic outcomes, such as the delivery of adjuvant chemotherapy [9, 10] or long-term survival $[11,12]$.

The objectives of the current study were to identify predictors of postoperative deterioration of performance status and cardiopulmonary complications, including parameters of preoperative physical functioning, in subjects treated with thoracoscopic lobectomy.

\section{Methods}

Study design and subjects

This prospective observational study was conducted between June 2005 and October 2012 at Shin-Kokura Hospital (Kitakyushu, Japan). Consecutive patients with preoperative stage I NSCLC who underwent thoracoscopic lobectomy were eligible for the current study. The indications for major pulmonary resection via thoracoscopic lobectomy were based on the standard criteria for an open thoracotomy, including a tumour of $\leqslant 6 \mathrm{~cm}$ in diameter [13], and a predicted postoperative (PPO) forced vital capacity of $>800 \mathrm{~mL} \cdot \mathrm{m}^{-2}$ or a forced expiratory volume in $1 \mathrm{~s}(\mathrm{FEV} 1)$ of $>600 \mathrm{~mL} \cdot \mathrm{m}^{-2}$ [14]. Patients were excluded if they had an Eastern Cooperative Oncology Group performance status [15] of 3-4, underwent pneumonectomy or were unable to walk independently at the time of admission. The hospital's institutional review board of clinical research ethically approved this study (approval number 2015-0005). Informed written consent was obtained for all subjects.

The present study had two primary outcomes: the incidence of deterioration of performance status after surgery and the incidence of postoperative cardiopulmonary complications. Performance status deterioration was defined by $\mathrm{a} \geqslant 1$-point increase in performance status score at postoperative assessment in comparison to the preoperative performance status score. The assessment was performed by certified physical therapists (M.I. and K.H.). The postoperative performance status was assessed on the day of the first outpatient visit after hospital discharge. Postoperative cardiopulmonary complications were defined as complications that occurred during the postoperative hospital stay. Any of the following postoperative events were considered in the analysis: respiratory failure requiring mechanical ventilation, pneumonia defined by typical clinical and laboratory features, atelectasis requiring bronchoscopy, prolonged air leakage for more than seven postoperative days, cardiac failure requiring inotropic support, unstable arrhythmia requiring medical treatment, bronchopleural fistula, empyema, chylothorax and massive subcutaneous emphysema. All complications were accurately graded according to the National Cancer Institute Common Terminology Criteria for Adverse Events version 3.0 [16], and events graded $\geqslant$ II were included in this study.

\section{Data collection and variables analysed}

Variables potentially related to the primary outcomes, including demographic, lung and physical functioning parameters and surgical and oncologic data, were collected prospectively. Preoperative pulmonary function tests, including spirometry and measurements of the diffusing capacity of the lung for carbon monoxide (DLCO), were performed by experienced technicians. The PPO values were obtained using the anatomic method, in which the preoperative value is multiplied by the fraction of the functional lung segments expected to remain after lobectomy. Therefore, the percentage of PPO (\%PPO) FEV 1 values were calculated using the following equation:

$$
\% \mathrm{PPO} \mathrm{FEV}_{1}=\mathrm{FEV}_{1} \% \text { predicted } \times(19-\text { number of segments resected }) / 19
$$

The \%PPO DLCO was calculated in the same manner. The parameters of the physical functioning, including the 6-min walk test (6MWT) and lower limb strength test, were assessed the day before surgery by the physical therapists. The 6MWT was performed according to the standardised guidelines [17]. Isometric lower limb strength was assessed using a hand-held dynamometer (Commander PowerTrack II; 
JTECH Medical, Salt Lake City, UT, USA). Knee extensor strength was measured with the patient in the sitting position and the knee positioned in approximately $90^{\circ}$ flexion. The dynamometer force pad was placed just proximal to the ankle joint and used to measure the force produced by quadriceps muscle contraction. All subjects were instructed to perform three maximal efforts, and the best result expressed in newton units was chosen and quantified in kilogram forces. Because muscle strength varies with body size and body mass normalisation represents a functional muscle strength measurement [18], the value was normalised to the direct percentage of body weight (\%BW).

\section{Surgical and perioperative management}

Preoperative staging included chest radiographic studies, bronchoscopy, brain magnetic resonance and bone scintigraphy. Thoracoscopic lobectomy, as previously reported [13], was performed under epidural catheter anaesthesia and general anaesthesia with dual-lumen endotracheal tubes in the absence of rib spreading. Postoperative chest pain was controlled by means of multimodal analgesia, including the provision of epidural or continuous intravenous analgesia and/or nonsteroidal anti-inflammatory drugs, which were titrated to maintain adequate pain control in order to achieve early mobilisation. According to the criteria of extubation in our previous report [13], the chest tube was routinely removed on postoperative day 1 . On the day before surgery, the subjects were informed about the role and contents of a structured postoperative pulmonary rehabilitation programme, which was performed by the physical therapists. As soon as the subjects were clinically stable and adequate pain control was confirmed, early postoperative mobilisation was begun on the morning of postoperative day 1 . On postoperative day 2, subjects with no complications were started on postoperative exercise training twice a day and continued until the time of hospital discharge, as previously reported [19].

\section{Statistical analysis}

The data are presented as absolute numbers and percentages for categorical variables and as the median and interquartile range (IQR) for continuous variables. A logistic regression analysis was used to determine the associations of the potential risk factors with the primary outcome variables and to estimate adjusted odds ratios and their 95\% confidence intervals. For the purpose of the regression analysis, all variables other than the surgical procedure were divided into two groups and analysed as binary data. The thresholds with regard to postoperative outcomes were made according to the most clinically relevant cut-off values [20-23]. A univariate analysis was first performed to identify any potential predictor variables. Variables with a p-value $<0.2$ according to a univariate analysis and those considered to be clinically relevant were included in the multivariate analysis to determine any independent predictors of the primary outcome variables. To avoid multicollinearity, only one variable was retained in a set of variables with a correlation coefficient $>0.9$. A stepwise selection procedure was used to identify variables with a p-value $<0.05$. The goodness-of-fit of the overall model was evaluated by the Hosmer-Lemeshow goodness-of-fit statistic, and the discriminatory capacity of the model was investigated using an area under the receiver operating characteristics curve (AUC) analysis. Furthermore, to increase the robustness of each model, the logistic regression analysis was validated by a bootstrap analysis with 1000 samples and an exact logistic regression analysis. A p-value $<0.05$ was considered to be statistically significant. Statistical analyses, other than the goodness-of-fit statistic, AUC analysis and the reliability tests, were performed using EZR (Saitama Medical Centre, Jichi Medical University, Saitama, Japan), which is a graphical user interface for R (The R Foundation for Statistical Computing, Vienna, Austria). More precisely, it is a modified version of $\mathrm{R}$ commander designed to add statistical functions frequently used in biostatistics [24]. All other statistical analyses were conducted using STATA statistical software (version 14; StataCorp, College Station, TX, USA).

\section{Results}

In the current study, $<10 \%$ of the eligible patients were excluded $(n=17)$, largely due to compromised lung function and/or the presence of significant comorbidities. The remaining 188 consecutive patients with preoperative stage I NSCLC who underwent thoracoscopic lobectomy were enrolled in the present study. The baseline characteristics of the subjects are shown in table 1. The distribution of preoperative lung function values is illustrated in figure 1, which shows that the subjects without comorbid chronic obstructive lung disease (COPD) had much higher lung function than the subjects with comorbid COPD. None of the subjects were converted from the thoracoscopic approach to open thoracotomy. Postoperative cardiopulmonary complications and the distribution of pathologic stages are shown in table 2 and figure 2, respectively. The rate of hospital mortality in this study was one subject $(0.5 \%)$ who underwent reoperation for a refractory air leak and developed respiratory failure requiring mechanical ventilation, finally dying from multiple organ failure on postoperative day 25. Given the small number of in-hospital deaths, mortality was not analysed separately, and this case was included as a patient with a worse outcome. 


\section{TABLE 1 Preoperative characteristics of study subjects}

\section{Subjects}

Age years

Females

Body mass index $\mathrm{kg} \cdot \mathrm{m}^{-2}$

Comorbidities

COPD

Charlson comorbidity index

0

1

3

$\geqslant 5$

ASA classification

II

$\geqslant 11$

mMRC dyspnoea scale

I

III

ECOG performance status

1

FEV $1 \%$ pred

D.co \% pred

Preoperative stage

IA

IB
188

$71(64-77)$

72 (38.3)

$22.1(21.0-24.8)$

149 (79.3)

66 (35.1)

$91(48.4)$

39 (20.7)

36 (19.1)

$13(6.9)$

7 (3.7)

2 (1.1)

25 (13.3)

89 (47.3)

74 (39.4)

154 (81.9)

28 (14.9)

6 (3.2)

$150(79.8)$

$36(19.1)$

2 (1.1)

99 (83-110)

96 (80-114)

134 (71.3)

54 (28.7)

Data are presented as $\mathrm{n}$, median (interquartile range) or $\mathrm{n}$ (\%). COPD: chronic obstructive pulmonary disease; ASA: American Society of Anesthesiologists; mMRC: modified Medical Research Council; ECOG: Eastern Cooperative Oncology Group; FEV1: forced expiratory volume in $1 \mathrm{~s}$; DLCO: diffusing capacity of the lung for carbon monoxide.

\section{Postoperative deterioration of the performance status}

$26(13.8 \%)$ of the 188 subjects showed deterioration in their postoperative performance status, the assessment of which was performed a median (IQR) 16 (14-20) days after surgery. Variables significantly associated with deterioration of performance status in the univariate analysis were DLCO \% pred and \% PPO, quadriceps muscle strength and pathologic stage (tables 3-5). In addition to these factors, the variables with $\mathrm{p}$-values $<0.2$ in the univariate analyses were considered for entry in the stepwise logistic
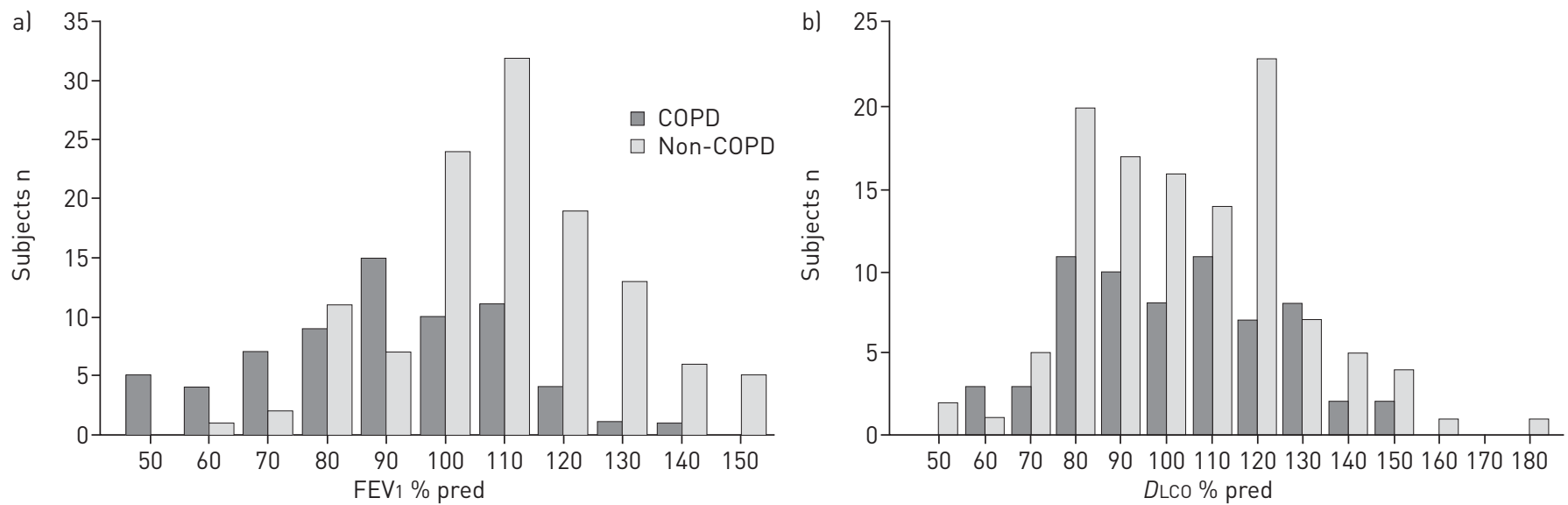

FIGURE 1 Distribution of a) forced expiratory volume in $1 \mathrm{~s}$ (FEV 1 ) and b) the diffusing capacity of the lung for carbon monoxide (DLco) in subjects with comorbid chronic obstructive pulmonary disease (COPD) ( $n=66)$ and without comorbid COPD ( $n=122$ ). 
TABLE 2 Postoperative cardiopulmonary complications occurring in 36 subjects after thoracoscopic lobectomy

$\begin{array}{lr}\text { Prolonged air leakage for }>\mathbf{7} \text { days } & 18(9.6) \\ \text { Atrial fibrillation requiring medical treatment } & 12(6.4) \\ \text { Atelectasis requiring bronchoscopy } & 8(4.3) \\ \text { Pneumonia } & 4(2.1) \\ \text { Massive subcutaneous emphysema } & 2(1.1) \\ \text { Chylothorax } & 1(0.5) \\ \text { Respiratory failure requiring mechanical ventilation } & 1(0.5)\end{array}$

Data are presented as $\mathrm{n}(\%) .{ }^{\#}$ : some subjects experienced more than one complication.

regression analysis. The multivariate analysis showed that DLCO $\%$ pred $(<80 \%)$, quadriceps muscle strength $(<40 \% \mathrm{BW})$ and pathologic stage ( $\geqslant$ IIA) were independent risk factors for deterioration of performance status after surgery (table 6). The final model fit the data well, and the discriminatory capacity was good (Hosmer-Lemeshow Chi-squared $=0.64, \mathrm{p}=0.73$ and $\mathrm{AUC}=0.84$, respectively). The predictive variables of the final model were confirmed to be reliable by reliability tests, including a bootstrap analysis and an exact logistic regression analysis.

\section{Postoperative cardiopulmonary complications}

Of the 188 subjects, postoperative cardiopulmonary complications were recorded in 36 cases and the incidence rate was $19.1 \%$ (table 2). The univariate analysis showed a significant association between postoperative cardiopulmonary complication and body mass index, comorbidity of COPD, the distance covered during 6MWT, amount of blood loss and pathologic stage (tables 3-5). The multivariate analysis was performed in the same manner as that of deterioration of performance status. As a result, significant predictors of postoperative cardiopulmonary complication were comorbidity of COPD, the distance covered during 6MWT $(<400 \mathrm{~m})$ and pathologic stage ( $\geqslant$ IIA) (table 6). Although the fit was marginal, the goodness-of-fit of the model was confirmed (Hosmer-Lemeshow Chi-squared=8.70, $\mathrm{p}=0.07$ ). The model also demonstrated a moderate discriminatory capacity $(\mathrm{AUC}=0.77)$. All of the variables in the final model were proven to be stable by the reliability tests.

\section{Discussion}

The major findings of our present study are as follows. 1) The incidence of postoperative deterioration of performance status and postoperative cardiopulmonary complication was $13.8 \%$ and $19.1 \%$, respectively, after thoracoscopic lobectomy; 2) DLCO \% pred, quadriceps muscle strength and pathologic stage were independent risk factors for deterioration of performance status; and 3) comorbidity of COPD, 6-min walking distance and pathologic stage were independent risk factors for postoperative cardiopulmonary complication. Clinical practice guidelines, which include a functional algorithm based on preoperative physiological evaluations, such as pulmonary function and exercise tests, were developed with the aim of determining whether patients should undergo curative surgical resection for lung cancer [20, 25].

FIGURE 2 Distribution of pathologic stages in postoperative subjects.

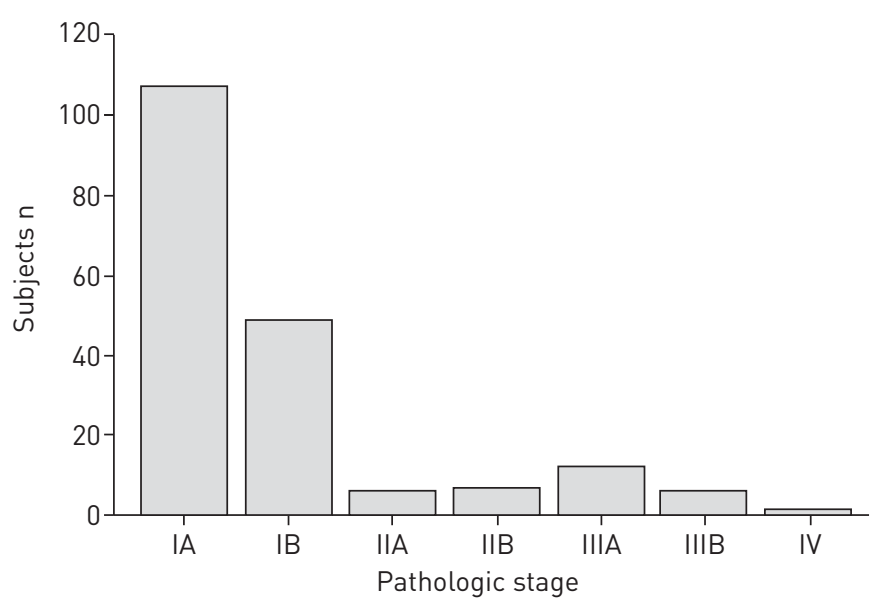


TABLE 3 Univariate analyses of demographic and clinical data in patients with or without postoperative deterioration of performance status and cardiopulmonary complications

\begin{tabular}{|c|c|c|c|c|c|c|c|c|}
\hline & \multicolumn{2}{|c|}{$\begin{array}{c}\text { Deterioration of } \\
\text { performance status }\end{array}$} & \multirow[t]{2}{*}{ OR $(95 \% \mathrm{CI})$} & \multirow[t]{2}{*}{ p-value } & \multicolumn{2}{|c|}{$\begin{array}{l}\text { Cardiopulmonary } \\
\text { complications }\end{array}$} & \multirow[t]{2}{*}{ OR $(95 \% \mathrm{CI})$} & \multirow[t]{2}{*}{ p-value } \\
\hline & Yes & No & & & Yes & No & & \\
\hline Age $\geqslant 70$ years & 16 (61.5) & 88 (54.3) & $1.34(0.57-3.14)$ & 0.49 & $23(63.9)$ & 81 (53.3) & $1.55(0.73-3.29)$ & 0.25 \\
\hline Females & $14(53.8)$ & 58 (35.8) & $2.08(0.91-4.76)$ & 0.083 & 12 (33.3) & 60 (39.5) & $0.77(0.36-1.64)$ & 0.50 \\
\hline Body mass index $<18.5 \mathrm{~kg} \cdot \mathrm{m}^{-2}$ & $4(15.4)$ & $16(9.9)$ & $1.66(0.51-5.42)$ & 0.40 & 8 (22.2) & $12(7.9)$ & $3.33(1.25-8.9)$ & 0.016 \\
\hline Charlson comorbidity index score $\geqslant 2$ & $9(34.6)$ & $49(30.2)$ & $1.29(0.54-3.13)$ & 0.56 & $10(27.8)$ & $48(31.6)$ & $0.83(0.37-1.85)$ & 0.64 \\
\hline ASA classification $\geqslant 3$ & $14(53.8)$ & 60 (37.0) & $1.98(0.86-4.57)$ & 0.11 & $19(52.8)$ & $55(36.2)$ & $1.97(0.94-4.10)$ & 0.07 \\
\hline mMRC dyspnoea scale grade $\geqslant I I$ & 5 (19.2) & 28 (17.3) & $1.18(0.41-3.40)$ & 0.76 & 7 (19.4) & $26(17.1)$ & $1.14(0.45-2.89)$ & 0.78 \\
\hline ECOG performance status $\geqslant 1$ & $7(26.9)$ & 31 (19.1) & $1.64(0.63-4.27)$ & 0.31 & $10(27.8)$ & $28(18.4)$ & $1.69(0.73-3.91)$ & 0.219 \\
\hline
\end{tabular}

Data are presented as $\mathrm{n}$ or $\mathrm{n}(\%)$, unless otherwise stated. COPD: chronic obstructive pulmonary disease; ASA: American Society of Anesthesiologists; mMRC: modified Medical Research Council; ECOG: Eastern Cooperative Oncology Group.

However, these guidelines were established according to evidence from a large body of literature assessing patients undergoing lung resection via the open thoracotomy approach. In a recent report, PATELLA et al. [6] developed a specific risk-adjusted morbidity model for thoracoscopic lobectomy. The current study was also conducted in patients who were undergoing thoracoscopic lobectomy and aimed to identify the risk factors for the postoperative outcome, which included measurements of physical function, such as functional capacity and quadriceps strength.

Although lower limb muscle strength has been used as the measured outcome in several recent randomised controlled trials of postoperative exercise interventions for lung cancer patients [26-28], this is the first study to demonstrate that poor muscle function is associated with the postoperative functional status. There are several possible reasons why the muscle function has predictive value. Lower limb muscle dysfunction is a major cause of exercise limitations in patients with chronic respiratory disease [29, 30], whose characteristics are mostly similar to those of patients with lung cancer. This could be related to the fact that such patients are unable to perform higher levels of postoperative exercise training. In addition, the peripheral muscle dysfunction noted in such patients may be attributable to systematic effects, such as deconditioning, inflammation and impaired energy balance [31]. In other words, because an individual's performance status is reflected in their general condition, poor muscle strength would predispose our patients to exhibit performance status deterioration. Therefore, the measurement of the quadriceps muscle strength using handheld dynamometry, which has the advantages of being both portable and inexpensive, is considered to be clinically useful for candidates for lung resection.

TABLE 4 Univariate analyses of lung and physical function data in patients with or without postoperative deterioration of performance status and cardiopulmonary complications

\begin{tabular}{|c|c|c|c|c|c|c|c|c|}
\hline & \multicolumn{2}{|c|}{$\begin{array}{l}\text { Deterioration of } \\
\text { performance status }\end{array}$} & \multirow[t]{2}{*}{ OR $(95 \% \mathrm{CI})$} & \multirow[t]{2}{*}{ p-value } & \multicolumn{2}{|c|}{$\begin{array}{l}\text { Cardiopulmonary } \\
\text { complications }\end{array}$} & \multirow[t]{2}{*}{ OR $(95 \% \mathrm{CI})$} & \multirow[t]{2}{*}{ p-value } \\
\hline & Yes & No & & & Yes & No & & \\
\hline FEV $1<80 \%$ pred & 9 (34.6) & 30 (18.5) & $2.31(0.94-5.69)$ & 0.07 & 10 (27.8) & $29(19.1)$ & $1.62(0.70-3.73)$ & 0.26 \\
\hline$D$ Lco $<80 \%$ pred & $13(50.0)$ & 32 (19.8) & $3.84(1.62-9.10)$ & 0.002 & 7 (19.4) & $38(25.0)$ & $0.68(0.28-1.68)$ & 0.40 \\
\hline PPO FEV $1<60 \%$ pred & $6(23.1)$ & $23(14.2)$ & $1.80(0.65-4.96)$ & 0.26 & $7(19.4)$ & $22(14.5)$ & $1.42(0.55-3.63)$ & 0.47 \\
\hline Desaturation during $6 \mathrm{MWT} \geqslant 4 \%$ & 4 (15.4) & $16(9.9)$ & $1.64(0.50-5.39)$ & 0.41 & $6(16.7)$ & $14(9.2)$ & $1.84(0.65-5.19)$ & 0.25 \\
\hline $\begin{array}{l}\text { Quadriceps muscle strength } \\
<40 \% \mathrm{BW}\end{array}$ & $17(65.4)$ & $59(36.4)$ & $3.79(1.48-9.68)$ & 0.005 & $17(47.2)$ & 59 (38.8) & $1.21(0.58-2.53)$ & 0.61 \\
\hline
\end{tabular}

Data are presented as $\mathrm{n}$ or $\mathrm{n}(\%)$, unless otherwise stated. $\mathrm{FEV}$ 1: forced expiratory volume in $1 \mathrm{~s}$; DLco: diffusing capacity of the lung for carbon monoxide; PPO: predicted postoperative; 6MWT: 6-min walk test; BW: bodyweight. 
TABLE 5 Univariate analyses of surgical and oncologic data in patients with or without postoperative deterioration of performance status and cardiopulmonary complications

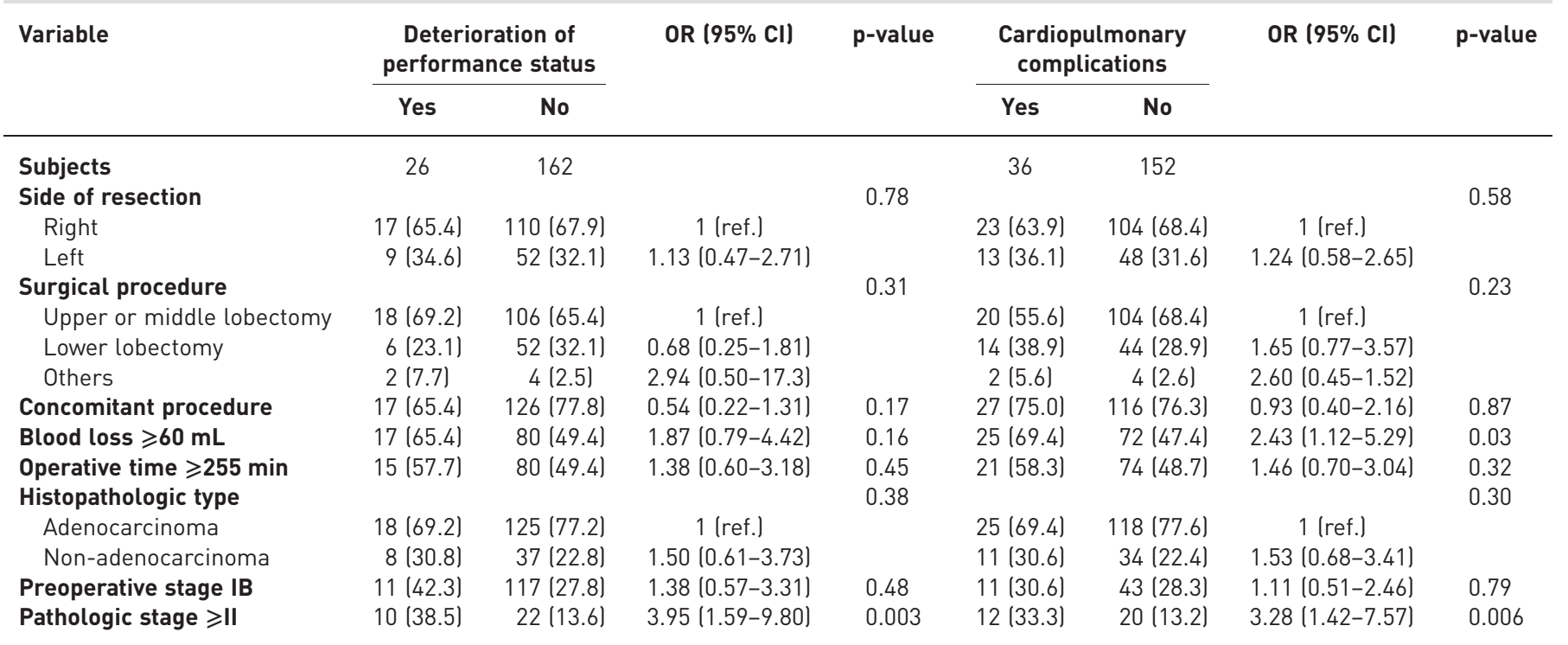

Data are presented as $\mathrm{n}$ or $\mathrm{n}(\%)$, unless otherwise stated.

This study demonstrated that the 6-min walking distance was an independent risk factor for postoperative complications. The available evidence, derived from a multivariate analysis, is insufficient to describe the role of the 6MWT in risk stratification before lung resection [20], whereas a recent retrospective study has documented that a shorter distance in the 6MWT is associated with an increased risk of postoperative complication [32]. In general, preoperative exercise capacity can be used to estimate the physiological reserve that may be available after surgery by stressing the whole oxygen delivery system. Formal cardiopulmonary exercise tests, requiring exercise equipment, an airflow transducer and gas analyser, are recommended to discriminate patients at risk of postoperative complications, because this test represents the most accurate assessment of maximum oxygen consumption [33, 34]. However, according to a survey report [35], the rate of implementation of such high-technology exercise tests is relatively low due to the lack of availability of technical resources. In contrast, the 6MWT is easy to use in this setting because of its simplicity and safety, with no equipment required [17]. Although the application of the 6MWT raises potential concerns about the so-called ceiling effect, due to the insufficient stress in patients with early-stage disease [36], this test may be clinically useful in patients considered for lung resection.

Unlike that observed in previous research $[2,7,8]$, DLCO \% pred failed to identify patients at risk for postoperative complications in the current study, although it was an independent predictor of performance status deterioration. In agreement with previous studies $[37,38]$, the comorbidity of COPD was identified

TABLE 6 Multivariate logistic regression analysis of the risk of postoperative deterioration of performance status and cardiopulmonary complications

OR $(95 \% \mathrm{CI})$

p-value

\begin{tabular}{|c|c|c|}
\hline \multicolumn{3}{|l|}{ Deterioration of performance status } \\
\hline$D$ Lco $<80 \%$ pred & $3.44(1.28-9.26)$ & 0.015 \\
\hline Quadriceps muscle strength $<40 \% \mathrm{BW}$ & $4.53(1.51-13.6)$ & 0.007 \\
\hline Pathologic stage $\geqslant 11$ & $9.50(2.99-30.2)$ & $<0.001$ \\
\hline \multicolumn{3}{|l|}{ Cardiopulmonary complications } \\
\hline COPD & $5.88(2.51-13.8)$ & $<0.001$ \\
\hline 6-min walking distance $<400 \mathrm{~m}$ & $4.04(1.60-10.2)$ & 0.031 \\
\hline Pathologic stage $\geqslant 11$ & $4.81(1.77-13.1)$ & 0.002 \\
\hline
\end{tabular}

$D\llcorner C O$ : diffusing capacity of the lung for carbon monoxide; BW: body weight; COPD: chronic obstructive pulmonary disease. 
to be an independent risk factor for postoperative complication. There are several possible explanations for this result: the presence of COPD may have contributed to prolonged air leakage during the period immediately after surgery due to the fragile nature of emphysematous lungs, since an increase in the right ventricular diastolic pressure is predictive of atrial arrhythmia [39]; and lung resection may cause temporary elevation in pulmonary artery afterload, resulting in an increased incidence of atrial fibrillation, particularly in patients with COPD.

Given that a greater pathologic stage was found to be a predictive factor for both deterioration of performance status and postoperative cardiopulmonary complication, cancer progression may affect long-term as well as short-term outcomes. NAKANISHI et al. [13] demonstrated that an advanced stage is associated with a higher incidence of complications, and another report by Licker et al. [37] showed that the diagnosis of earlier cancer stages probably contributes to better short-term outcomes. This suggests that patients with a greater pathologic stage are more likely to undergo more extensive surgical stress, resulting in a worse postoperative outcome than that observed in subjects at an earlier stage.

We recognise that there are some important limitations to this analysis. First, the main limitations of this study are its retrospective nature and the fact that only subjects treated at a single institution were examined. Therefore, we should take into account the inherent biases in patient selection and experience of the surgeons. We attempted to minimise these biases by limiting our analysis to patients with preoperative stage I NSCLC only, who were deemed to undergo simple lobectomy without an extended procedure. In addition, the hospital and surgeon volume of our institution is high (defined as $>20$ cases per year [40]), which may lessen biases regarding surgical techniques and postoperative management. Second, the small sample size of this study might have interfered with the reliability of the regression analysis. However, this issue was partially overcome by the bootstrap and exact logistic analyses, which showed the robustness of the models. Third, several potential confounding variables may influence the postoperative outcomes. Fourth, timing of the reassessment of the performance status varied according to the timing of the individual's hospital discharge; this might have reduced the quality of these data. In addition, the lack of blinding of the performance status assessors had the potential to introduce considerable bias into the study results. Finally, performance status deterioration might have a negative impact on the long-term outcomes because patients who still have a poor functional status will be unable to commence adjuvant chemotherapy. Nonetheless, our study did not investigate the long-term outcomes.

In conclusion, our data suggest that, in addition to a greater pathologic stage, lower DLCO and comorbid COPD, poor physical function was associated with worse short-term outcomes after thoracoscopic lobectomy. A possible clinical implication of this study is that the assessment of quadriceps muscle strength and 6MWT, which can be used widely in various clinical settings, may therefore be a useful and effective method for identifying risk factors for short-term outcomes in cases of thoracoscopic lobectomy. Further prospective controlled trials are needed to confirm these findings.

\section{Acknowledgements}

We would like to acknowledge all subjects who kindly agreed to participate in the study. We have presented the results of this study previously, at the World Confederation for Physical Therapy Congress in Singapore in May 2015.

\section{References}

1 Paul S, Altorki NK, Sheng S, et al. Thoracoscopic lobectomy is associated with lower morbidity than open lobectomy: a propensity-matched analysis from the STS database. J Thorac Cardiovasc Surg 2010; 139: 366-378.

2 Burt BM, Kosinski AS, Shrager JB, et al. Thoracoscopic lobectomy is associated with acceptable morbidity and mortality in patients with predicted postoperative forced expiratory volume in 1 second or diffusing capacity for carbon monoxide less than 40\% of normal. J Thorac Cardiovasc Surg 2014; 148: 19-28.

3 Stephens N, Rice D, Correa A, et al. Thoracoscopic lobectomy is associated with improved short-term and equivalent oncological outcomes compared with open lobectomy for clinical stage I non-small-cell lung cancer: a propensity-matched analysis of 963 cases. Eur J Cardiothorac Surg 2014; 46: 607-613.

4 Yang J, Xia Y, Yang Y, et al. Risk factors for major adverse events of video-assisted thoracic surgery lobectomy for lung cancer. Int J Med Sci 2014; 11: 863-869.

5 Villamizar NR, Darrabie M, Hanna J, et al. Impact of $\mathrm{T}$ status and $\mathrm{N}$ status on perioperative outcomes after thoracoscopic lobectomy for lung cancer. J Thorac Cardiovasc Surg 2013; 145: 514-520.

6 Patella M, Sandri A, Pompili C, et al. Real-time monitoring of a video-assisted thoracoscopic surgery lobectomy programme using a specific cardiopulmonary complications risk-adjusted control chart. Eur J Cardiothorac Surg 2016; 49: 1070-1074.

7 Berry MF, Villamizar-Ortiz NR, Tong BC, et al. Pulmonary function tests do not predict pulmonary complications after thoracoscopic lobectomy. Ann Thorac Surg 2010; 89: 1044-1051.

8 Ceppa DP, Kosinski AS, Berry MF, et al. Thoracoscopic lobectomy has increasing benefit in patients with poor pulmonary function: a Society of Thoracic Surgeons Database analysis. Ann Surg 2012; 256: 487-493.

9 Lee JG, Cho BC, Bae MK, et al. Thoracoscopic lobectomy is associated with superior compliance with adjuvant chemotherapy in lung cancer. Ann Thorac Surg 2011; 91: 344-348. 
10 Teh E, Abah U, Church D, et al. What is the extent of the advantage of video-assisted thoracoscopic surgical resection over thoracotomy in terms of delivery of adjuvant chemotherapy following non-small-cell lung cancer resection? Interact Cardiovasc Thorac Surg 2014; 19: 656-660.

11 Rueth NM, Parsons HM, Habermann EB, et al. The long-term impact of surgical complications after resection of stage I nonsmall cell lung cancer: a population-based survival analysis. Ann Surg 2011; 254: 368-374.

12 Andalib A, Ramana-Kumar AV, Bartlett G, et al. Influence of postoperative infectious complications on long-term survival of lung cancer patients: a population-based cohort study. J Thorac Oncol 2013; 8: 554-561.

13 Nakanishi R, Fujino Y, Yamashita T, et al. A prospective study of the association between drainage volume within 24 hours after thoracoscopic lobectomy and postoperative morbidity. J Thorac Cardiovasc Surg 2009; 137: 1394-1399.

14 Uramoto H, Nakanishi R, Fujino Y, et al. Prediction of pulmonary complications after a lobectomy in patients with non-small cell lung cancer. Thorax 2001; 56: 59-61.

15 Oken MM, Creech RH, Tormey DC, et al. Toxicity and response criteria of the Eastern Cooperative Oncology Group. Am J Clin Oncol 1982; 5: 649-655.

16 National Cancer Institute, Cancer Therapy Evaluation Program. Common Terminology Criteria for Adverse Events (CTCAE) v3.0. http://ctep.cancer.gov/protocolDevelopment/electronic_applications/docs/ctcaev3.pdf Date last accessed: July 10, 2011. Date last updated: August 9, 2006.

17 ATS Committee on Proficiency Standards for Clinical Pulmonary Function Laboratories. ATS statement: guidelines for the six-minute walk test. Am J Respir Crit Care Med 2002; 166: 111-117.

18 Jaric S. Muscle strength testing: use of normalisation for body size. Sports Med 2002; 32: 615-631.

19 Irie M, Nakanishi R, Hamada K, et al. Perioperative short-term pulmonary rehabilitation for patients undergoing lung volume reduction surgery. COPD 2011; 8: 444-449.

20 Brunelli A, Kim AW, Berger KI, et al. Physiologic evaluation of the patient with lung cancer being considered for resectional surgery: diagnosis and management of lung cancer, 3rd ed: American College of Chest Physicians evidence-based clinical practice guidelines. Chest 2013; 143: Suppl. 5, e166S-e190S.

21 Shannon VR. Role of pulmonary rehabilitation in the management of patients with lung cancer. Curr Opin Pulm Med 2010; 16: 334-339.

22 Brunelli A. Risk assessment for pulmonary resection. Semin Thorac Cardiovasc Surg 2010; 22: 2-13.

23 Nakanishi R, Yamashita T, Oka S. Video-assisted thoracic surgery lobectomy for non-small cell lung cancer in patients with a Charlson comorbidity index score of two or more. J Thorac Oncol 2010; 5: 56-61.

24 Kanda Y. Investigation of the freely available easy-to-use software 'EZR' for medical statistics. Bone Marrow Transplant 2013; 48: 452-458.

25 Brunelli A, Charloux A, Bolliger CT, et al. ERS/ESTS clinical guidelines on fitness for radical therapy in lung cancer patients (surgery and chemo-radiotherapy). Eur Respir J 2009; 34: 17-41.

26 Arbane G, Tropman D, Jackson D, et al. Evaluation of an early exercise intervention after thoracotomy for non-small cell lung cancer (NSCLC), effects on quality of life, muscle strength and exercise tolerance: randomised controlled trial. Lung Cancer 2011; 71: 229-234.

27 Arbane G, Douiri A, Hart N, et al. Effect of postoperative physical training on activity after curative surgery for non-small cell lung cancer: a multicentre randomised controlled trial. Physiotherapy 2014; 100: 100-107.

28 Edvardsen E, Skjønsberg OH, Holme I, et al. High-intensity training following lung cancer surgery: a randomised controlled trial. Thorax 2015; 70: 244-250.

29 Gosselink R, Troosters T, Decramer M. Peripheral muscle weakness contributes to exercise limitation in COPD. Am J Respir Crit Care Med 1996; 153: 976-980.

30 Seymour JM, Spruit MA, Hopkinson NS, et al. The prevalence of quadriceps weakness in COPD and the relationship with disease severity. Eur Respir J 2010; 36: 81-88.

31 Maltais F, Decramer M, Casaburi R, et al. An official American Thoracic Society/European Respiratory Society statement: update on limb muscle dysfunction in chronic obstructive pulmonary disease. Am J Respir Crit Care Med 2014; 189: e15-e62.

32 Marjanski T, Wnuk D, Bosakowski D, et al. Patients who do not reach a distance of $500 \mathrm{~m}$ during the 6-min walk test have an increased risk of postoperative complications and prolonged hospital stay after lobectomy. Eur J Cardiothorac Surg 2015; 47: e213-e219.

33 Benzo R, Kelley GA, Recchi L, et al. Complications of lung resection and exercise capacity: a meta-analysis. Respir Med 2007; 101: 1790-1797.

34 Loewen GM, Watson $\mathrm{D}$, Kohman $\mathrm{L}$, et al. Preoperative exercise $\mathrm{VO}_{2}$ measurement for lung resection candidates: results of Cancer and Leukemia Group B Protocol 9238. J Thorac Oncol 2007; 2: 619-625.

35 Charloux A, Brunelli A, Bolliger CT, et al. Lung function evaluation before surgery in lung cancer patients: how are recent advances put into practice? A survey among members of the European Society of Thoracic Surgeons (ESTS) and of the Thoracic Oncology Section of the European Respiratory Society (ERS). Interact Cardiovasc Thorac Surg 2009; 9: 925-931.

36 Jones LW, Eves ND, Haykowsky M, et al. Cardiorespiratory exercise testing in clinical oncology research: systematic review and practice recommendations. Lancet Oncol 2008; 9: 757-765.

37 Licker MJ, Widikker I, Robert J, et al. Operative mortality and respiratory complications after lung resection for cancer: impact of chronic obstructive pulmonary disease and time trends. Ann Thorac Surg 2006; 81: 1830-1837.

38 Pompili C, Brunelli A, Refai M, et al. Does chronic obstructive pulmonary disease affect postoperative quality of life in patients undergoing lobectomy for lung cancer? A case-matched study. Eur J Cardiothorac Surg 2010; 37: 525-530.

39 Lindgren L, Lepäntalo $\mathrm{M}$, von Knorring $\mathrm{J}$, et al. Effect of verapamil on right ventricular pressure and atrial tachyarrhythmia after thoracotomy. Br J Anaesth 1991; 66: 205-211.

40 Park HS, Detterbeck FC, Boffa DJ, et al. Impact of hospital volume of thoracoscopic lobectomy on primary lung cancer outcomes. Ann Thorac Surg 2012; 93: 372-379. 